\title{
Knowledge and Practices in the Diagnosis and Treatment of Corneal Infections by Nepalese Pharmaceutical Shop Workers
}

\author{
Sadhan Bhandari, ${ }^{1} \dagger$ John M. Nesemann, ${ }^{2 *} \dagger$ Ram Prasad Kandel, ${ }^{3}$ Bimal Poudyal, ${ }^{1}$ Gopal Bhandari, ${ }^{1}$ Raghunandan Byanju, ${ }^{1}$ \\ Riju Shrestha, ${ }^{4}$ David A. Ramirez, ${ }^{5}$ Kieran S. O'Brien, ${ }^{2}$ Thomas M. Lietman, ${ }^{3,6}$ and Jeremy D. Keenan ${ }^{3,6}$ \\ ${ }^{1}$ Bharatpur Eye Hospital, Bharatpur, Nepal; ${ }^{2}$ Francis I. Proctor Foundation, University of California San Francisco, San Francisco, California; ${ }^{3}$ Seva \\ Foundation, Berkeley, California; ${ }^{4}$ Department of Epidemiology, Gilling School of Public Health, University of North Carolina at Chapel Hill, Chapel \\ Hill, North Carolina; ${ }^{5}$ Department of Ophthalmology and Visual Sciences, University of lowa, lowa City, lowa; ${ }^{6}$ Department of Ophthalmology, \\ University of California San Francisco, San Francisco, California
}

\begin{abstract}
Medical shops in Nepal are a main point of treatment for many diseases including ophthalmic conditions. We sought to evaluate pharmaceutical shop worker knowledge of corneal ulcers and abrasions. A pharmaceutical shop worker from each of 117 different pharmacies surrounding Bharatpur, Nepal, was presented four different eye photographs (i.e., corneal ulcer, corneal abrasion, conjunctivitis, and a normal eye) and asked about diagnosis and management. Of 117 participants, $86(74 \%)$ identified conjunctivitis correctly but few were able to identify corneal abrasion (50/ $117 ; 43 \%)$ or corneal infection $(47 / 117 ; 40 \%)$. When presented with an illustrated diagram of potential medications to dispense, 15 (13\%) participants chose a topical medication containing a corticosteroid for the corneal abrasion and 25 $(21 \%)$ did so for the corneal ulcer. The appropriate use of corticosteroids for external eye infections is an important topic for additional training, given the potential for these medications to worsen corneal abrasions and ulcers.
\end{abstract}

\section{INTRODUCTION}

In developing countries a large proportion of the population lives in rural areas where healthcare facilities are scarce. ${ }^{1}$ Many people living on the Indian subcontinent use community pharmacies or medical shops as their primary site of health care, including for eye conditions. ${ }^{2,3}$ It is often easier to access a medical shop, where workers can advise a treatment course and dispense medications without a doctor's prescription. However, the knowledge base of pharmaceutical shop workers has not been well characterized, especially as it relates to management of eye disease. For example, current regulations in Nepal stipulate that pharmacists or pharmacy assistants have completed a 3-year pharmacy program and be registered with the Nepali Pharmacy Council but do not require any specific ophthalmic training.

In the present study, we administered a questionnaire to pharmaceutical shop workers in Nepal to determine their familiarity with the diagnosis and management of common eye conditions. We were especially interested in how frequently workers would advise topical corticosteroids for corneal ulcers or abrasions because such a recommendation could worsen the infection and cause irreversible deterioration of vision. We hypothesized that individuals with pharmaceutical and eye hospital training would be more likely to correctly identify and manage corneal infections.

\section{METHODS}

Study design. A cross-sectional study was conducted in June and July of 2017. All medical shops within the Village Integrated Eye Worker Trial I (VIEW I) study area were eligible for participation. ${ }^{4}$ Medical shops were identified by asking residents in each community; the intent was to capture all medical shops in the study area. Consenting workers present at the time of survey administration had their demographic,

\footnotetext{
*Address correspondence to John Nesemann, Francis I. Proctor Foundation, 113 South Ferry Dr., Lake Mills, WI 53551. E-mail: jnesemann@mednet.ucla.edu

†These authors contributed equally to the work.
}

educational, and training information recorded. They were then presented four illustrated clinical vignettes and asked the same set of questions for each scenario, focusing on diagnosis and management. The four scenarios were corneal ulcer, corneal abrasion, allergic conjunctivitis, and a normal eye examination (Supplemental Figure 1). The allergic conjunctivitis scenario was included as a type of control because corticosteroids are a major treatment modality for this condition but generally should not be prescribed for corneal abrasions or ulcers. Questions about treatment were first asked in an open-ended format, then in a multiple-choice format with an accompanying diagram of widely available ophthalmic medications.

Statistical considerations. There were two exposures of interest: 1) the amount of pharmaceutical training, categorized as no formal pharmacology training versus some training (Table 1) and 2) participation in a 1-day training workshop for pharmaceutical shop workers conducted by Bharatpur Eye Hospital. Workshops covered general eye anatomy, the differential diagnosis of a red eye, and the consequences of corticosteroid use. There had been three training sessions in the study area, with the most recent offered 3 years before survey administration. The main outcomes of interest were 1) whether the condition was correctly diagnosed and 2) whether corticosteroids were recommended for corneal ulcers or abrasions in the free response question. Logistic regression models were constructed for each outcome and adjusted for years of work experience. All analyses were considered exploratory, with a significance level of 0.05 for each. Analyses were performed with $R$ version 3.6.0. ${ }^{5}$

Ethics. The study received ethical approval from the University of California, San Francisco; Nepal Netra Jyoti Sangh; and the Nepal Health Research Council. Oral consent for participants was approved by the ethics committees and obtained for all participants; no stipend was provided. The research adhered to the guidelines outlined in the Declaration of Helsinki.

\section{RESULTS}

In total, 117 medical shops were identified in the VIEW I study area. A pharmaceutical shop worker from each pharmacy participated in the study; there were no refusals. Of the 
TABLE 1

Demographic information of 117 pharmaceutical shop workers in Chitwan and Nawalparasi districts, Nepal

\begin{tabular}{|c|c|}
\hline Characteristic & $N(\%)$ or mean $( \pm \mathrm{SD})$ \\
\hline Male & 93 (79.5\%) \\
\hline Age (years) & $39.2( \pm 11.6)$ \\
\hline Received training from eye hospital ${ }^{*}$ & $21(17.9 \%)$ \\
\hline \multicolumn{2}{|l|}{ Pharmaceutical training } \\
\hline \multicolumn{2}{|l|}{ Some } \\
\hline Medical degree & $2(1.7 \%)$ \\
\hline Pharmacy degree & $9(7.7 \%)$ \\
\hline Homeopathic pharmacy degree & $1(0.9 \%)$ \\
\hline Health assistant & $8(6.8 \%)$ \\
\hline Community medical assistant & $62(53.0 \%)$ \\
\hline Staff nurse & $1(0.9 \%)$ \\
\hline Government workshop† & $12(10.3 \%)$ \\
\hline \multicolumn{2}{|l|}{ None } \\
\hline Medical technician (Bachelor's) & $1(0.9 \%)$ \\
\hline Laboratory technician & $2(1.7 \%)$ \\
\hline Radiology technician & $1(0.9 \%)$ \\
\hline Ayurvedic health worker & $2(1.7 \%)$ \\
\hline Auxiliary nurse midwife & $2(1.7 \%)$ \\
\hline Nepali sena training $\ddagger$ & $1(0.9 \%)$ \\
\hline None & $13(11.1 \%)$ \\
\hline \multicolumn{2}{|c|}{$\begin{array}{l}\text { *Eye training offered by Bharatpur Eye Hospital consisting of a single day of training } \\
\text { discussing general eye anatomy, differential diagnosis of a red eye, and consequences of } \\
\text { steroid use. There had been three trainings before the study, with the last one taking place } \\
\text { three years before survey administration. } \\
\text { Three-day government-sponsored training program for pharmaceutical shop workers } \\
\text { without formal pharmaceutical training; these have been discontinued since } 2010 \text {. } \\
\ddagger \text { Training within the Nepalese army, involving first aid and basic primary care training. }\end{array}$} \\
\hline
\end{tabular}

117 respondents, the mean age was 39 years (SD 11.6) and 93 (79.5\%) were male; 95 (81.2\%) workers were classified as having some pharmaceutical training, and 21 (17.9\%) had participated in an eye hospital workshop (Table 1).

Diagnosis and management responses for each eye condition are summarized in Table 2. Among the 117 participants, 86 (74\%) correctly identified allergic conjunctivitis, but few identified corneal abrasion (50/117; 43\%) or ulcer (47/117; 40\%). When asked a free-response question, most workers answered they would dispense a topical antibiotic for the corneal abrasion (96/ $117 ; 82 \%)$ or ulcer $(94 / 117 ; 80 \%)$, and a few responded they would dispense a topical corticosteroid $(7 / 117 ; 6 \%$ and $6 / 117$; $5 \%$, respectively). However, when given a multiple-choice question, 15 of the 117 (13\%) respondents indicated they would dispense a topical corticosteroid for a corneal abrasion, and $25(21 \%)$ for a corneal ulcer. Corticosteroids were almost always selected as part of a combination antibiotic-corticosteroid eye drop (Supplemental Table 1). When asked for further recommendations in an open-ended way, most workers responded they would recommend a visit to an eye care specialist, with the highest proportion recommending this for the corneal ulcer (91/ $117 ; 78 \%)$ and abrasion $(81 / 117 ; 69 \%)$ scenarios.

We explored the data to assess whether those with more training were more likely to respond with a correct diagnosis and less likely to recommend a corticosteroid for the corneal ulcer and abrasion scenarios. As shown in Table 3, no statistically significant associations were found between either 1) having participated in a pharmacologic training program or 2) having participated in a Bharatpur Eye Hospital workshop and either of the outcomes of interest (i.e., correct diagnosis and recommendation of corticosteroid).

\section{DISCUSSION}

This study found that a minority of pharmaceutical shop workers could correctly diagnosis a corneal ulcer or corneal abrasion when presented with an illustrated vignette. Most respondents would appropriately dispense a topical antibiotic for each of these conditions; few would include a topical corticosteroid. Neither pharmacologic training nor participation in an eye hospital workshop significantly changed the odds of a correct diagnosis or of dispensing a topical corticosteroid.

Less than half of respondents correctly identified photographs of corneal ulcers and abrasions, and a majority (74\%) correctly diagnosed allergic conjunctivitis. These results are similar to those of Burn et al. ${ }^{6}$ who found that $27 \%$ of Nepali primary healthcare workers were able to diagnose corneal ulcers and $83 \%$ conjunctivitis. These studies suggest a lack of ophthalmic knowledge among the lower level health workers who provide a great deal of medical care in Nepal. This study did not find that an eye hospital workshop or more pharmaceutical training significantly increased the likelihood of correctly diagnosing an ulcer or abrasion, which we speculate could have been because of a training program that was of inadequate duration or that took place too long ago, or alternatively because of inadequate statistical power in this study. Nonetheless, the gaps in knowledge highlighted in this survey identify topics that could be included in future continuing medical education programs directed at pharmaceutical shop workers or primary healthcare workers, especially given the high prevalence of corneal abrasions and ulcers in Nepal. ${ }^{7}$

Despite a minority correctly identifying the two conditions, the vast majority of workers correctly recommended a topical antibiotic for the corneal ulcer and abrasion scenarios. Two-thirds would also dispense topical antibiotics for the allergic conjunctivitis vignette, and almost a third would do so for the normal eye. Although we do not fault workers for considering an infectious etiology for the conjunctivitis vignette, the relatively high use of antibiotics for the normal eye suggests that antibiotics may be recommended for most eye complaints, regardless of diagnosis. This finding is in line with other reports detailing excessive oral antibiotic prescriptions in pharmacies in Nepal and in low- and middle-income countries more generally. ${ }^{8-10}$

The percentage of pharmaceutical workers prescribing corticosteroids for corneal abrasions or ulcers was low, although this

TABLE 2

Survey responses of 117 pharmaceutical shop workers in Chitwan and Nawalparasi districts, Nepal

\begin{tabular}{|c|c|c|c|c|c|c|c|}
\hline \multirow[b]{2}{*}{ Photograph } & \multirow[b]{2}{*}{ Correctly identified } & \multirow[b]{2}{*}{ Most common misdiagnosis } & \multicolumn{2}{|c|}{ Would dispense topical antibiotic } & \multicolumn{2}{|c|}{ Would dispense any topical steroid } & \multirow[b]{2}{*}{ Would refer } \\
\hline & & & Free response & Multiple choice & Free response & Multiple choice & \\
\hline Corneal ulcer & $47(40 \%)$ & Conjunctivitis (32; 27\%) & $94(80 \%)$ & $98(84 \%)$ & $6(5 \%)$ & $25(21 \%)$ & $91(78 \%)$ \\
\hline Corneal abrasion & $50(43 \%)$ & Corneal ulcer $(28 ; 24 \%)$ & $96(82 \%)$ & $88(75 \%)$ & $7(6 \%)$ & $15(13 \%)$ & $81(69 \%)$ \\
\hline Allergic conjunctivitis & $86(74 \%)$ & Corneal ulcer $(14 ; 12 \%)$ & $80(68 \%)$ & $78(67 \%)$ & $28(23 \%)$ & $30(26 \%)$ & $67(57 \%)$ \\
\hline Normal eye & $43(37 \%)$ & Do not know (52; 46\%) & $34(29 \%)$ & $41(35 \%)$ & $6(5 \%)$ & $8(7 \%)$ & $55(47 \%)$ \\
\hline
\end{tabular}


TABLE 3

Relationship between training and diagnosis/management

\begin{tabular}{|c|c|c|c|c|c|c|c|c|c|c|}
\hline \multirow[b]{3}{*}{ Form of training } & \multirow{2}{*}{\multicolumn{2}{|c|}{$\begin{array}{c}\text { Training } \\
\text { Dx }\end{array}$}} & \multirow{2}{*}{\multicolumn{2}{|c|}{$\begin{array}{c}\text { No training } \\
\text { Dx }\end{array}$}} & \multirow{3}{*}{$\begin{array}{c}\text { OR for correct } \\
\text { diagnosis }(95 \% \mathrm{Cl})\end{array}$} & \multirow{2}{*}{\multicolumn{2}{|c|}{$\begin{array}{l}\text { Training } \\
\text { Steroids }\end{array}$}} & \multirow{2}{*}{\multicolumn{2}{|c|}{$\begin{array}{c}\text { No training } \\
\text { Steroids }\end{array}$}} & \multirow{3}{*}{$\begin{array}{l}\text { OR for dispensing } \\
\text { corticosteroid }(95 \% \mathrm{Cl})\end{array}$} \\
\hline & & & & & & & & & & \\
\hline & + & - & + & - & & + & - & + & - & \\
\hline \multicolumn{11}{|l|}{ Corneal ulcer } \\
\hline Pharmacology & 38 & 57 & 9 & 13 & $0.90(0.34-2.42)$ & 6 & 89 & 0 & 22 & $3.34(0.37-444.2)$ \\
\hline Eye hospital & 7 & 14 & 40 & 56 & $0.57(0.19-1.56)$ & 0 & 21 & 6 & 90 & $0.32(0.01-3.02)$ \\
\hline \multicolumn{11}{|l|}{ Corneal abrasion } \\
\hline Pharmacology & 43 & 52 & 7 & 15 & $1.79(0.69-5.06)$ & 4 & 91 & 3 & 19 & $0.28(0.06-1.52)$ \\
\hline Eye hospital & 9 & 12 & 41 & 55 & $1.02(0.38-2.68)$ & 1 & 20 & 6 & 90 & $0.77(0.04-5.14)$ \\
\hline
\end{tabular}

The exposures of interest are participation in pharmacology training or an eye hospital workshop: the outcomes are whether a correct diagnosis was provided and whether a topical corticosteroid would be dispensed, with numbers given for both the corneal ulcer and corneal abrasion photographs.

depended on how the question was asked. Few respondents suggested a corticosteroid when asked in an open-ended fashion but more respondents recommended an eye drop containing a corticosteroid when presented with a list of possible medication choices. These eye drops were almost always combination drops consisting of an antibiotic and corticosteroid, which are widely available throughout Nepal and are very familiar to any medical shop worker. Although arguably better to recommend a combination eye drop for corneal ulcers or abrasions than a corticosteroid eye drop alone, the provision of corticosteroids in any form by nonophthalmic personnel for either of these conditions is something that should probably be avoided, especially because corticosteroids are contraindicated in fungal keratitis and Nepal has a relatively high prevalence of fungal corneal ulcers. ${ }^{11}$

The present study has several limitations. Although the inclusion of all medical shops in the study area increases its generalizability, we interviewed only a single worker from each medical shop, and this person may or may not have been representative of the other workers at the shop. Moreover, the generalizability of the study outside this region of Nepal is uncertain. The use of a single photograph and limited information in the clinical vignette cannot replicate an in-person history and physical examination. The training variables assessed in this study were elicited by self-report and dichotomized, which could have resulted in misclassification bias and reduced statistical power, respectively.

In conclusion, most pharmaceutical shop workers recommended appropriate management for corneal ulcers and abrasions, even if they were unable to diagnose the specific conditions. Educational gaps identified in this survey included the ability to correctly diagnose corneal abrasions and corneal ulcers and knowledge that these conditions should not be treated with eye drops containing a corticosteroid.

Received May 28, 2020. Accepted for publication June 16, 2020.

Published online July 20, 2020.

Note: Supplemental table and figure appear at www.ajtmh.org.

Financial support: The study was supported by grants from That Man May See, the Fortisure Foundation, the Harper-Inglis Memorial for Eye Research, the Peierls Foundation, the Alta California Eye Research Foundation, the Bofinger Glaucoma Research Fund, and Research to Prevent Blindness. This project was supported by the National Eye Institute and the Fogarty International Center of the National Institutes of Health (NIH) under Award Number D43TW009343 as well as the University of California Global Health Institute (UCGHI) in the form of a Fogarty grant to J. N.

Authors' addresses: Sadhan Bhandari, Bimal Poudyal, Gopal Bhandari, and Raghunandan Byanju, Bharatpur Eye Hospital, Bharatpur,
Nepal, E-mails: bhandarisadhan123@gmail.com, bpoudyal@ntc.net.np, uniteforsightgopal@gmail.com, and raghunandan.byanju@gmail.com. John M. Nesemann, Geffen School of Medicine (Medical Student), University of California Los Angeles, Los Angeles, CA, E-mail: jnesemann@ mednet.ucla.edu. Ram Prasad Kandel, Seva Foundation, Berkeley, CA, E-mail: seva.kandel@ntc.net.np. Riju Shrestha, Department of Epidemiology, Gilling School of Public Health, University of North Carolina at Chapel Hill, Chapel Hill, NC, E-mail: riju@live.unc.edu. David A. Ramirez, Department of Ophthalmology and Visual Sciences, University of lowa, lowa City, IA, E-mail: david-ramirez@uiowa.edu. Kieran S. O'Brien, Thomas M. Lietman, and Jeremy D. Keenan, Francis I Proctor Foundation, University of California San Francisco, San Francisco, CA, E-mails: kieran.obrien@ucsf.edu, tom.lietman@ucsf.edu, and jeremy.keenan@ ucsf.edu.

\section{REFERENCES}

1. Ministry of Health - MOH/Nepal, New ERA/Nepal, ICF, 2017. Nepal Demographic and Health Survey 2016. Kathmandu, Nepal: MOH/Nepal, New ERA, and ICF.

2. Ansari $M, 2017$. Evaluation of community pharmacies regarding dispensing practices of antibiotics in two districts of central Nepal. PLoS One 12: e0183907.

3. Kafle KK, Gartoulla RP, Pradhan YM, Shrestha AD, Karkee SB, Quick JD, 1992. Drug retailer training: experiences from Nepal. Soc Sci Med 35: 1015-1025.

4. O'Brien KS et al.; Village-Integrated Eye Worker Trial Group, 2018. Village-integrated eye worker trial (VIEW): rationale and design of a cluster-randomised trial to prevent corneal ulcers in resource-limited settings. BMJ Open 8: e021556.

5. Team RC, 2018. R: A Language and Environment for Statistical Computing. Vienna, Austria: Foundation for Statistical Computing.

6. Burn H, Puri L, Roshan A, Singh SK, Burton MJ, 2020. Primary eye care in eastern Nepal. Ophthalmic Epidemiol 27: 165-176.

7. Upadhyay MP, Karmacharya PC, Koirala S, Tuladhar NR, Bryan LE, Smolin G, Whitcher JP, 1991. Epidemiologic characteristics, predisposing factors, and etiologic diagnosis of corneal ulceration in Nepal. Am J Ophthalmol 111: 92-99.

8. Nepal A, Hendrie D, Robinson S, Selvey LA, 2019. Survey of the pattern of antibiotic dispensing in private pharmacies in Nepal. BMJ Open 9: e032422.

9. WHO, 2009. Medicines Use in Primary Care in Developing and Transitional Countries: Fact Book Summarizing Results from Studies Reported between 1990 and 2006. Geneva, Switzerland: World Health Organization.

10. Asbell PA, Sanfilippo CM, Pillar CM, DeCory HH, Sahm DF, Morris TW, 2015. Antibiotic resistance among ocular pathogens in the United States: five-year results from the antibiotic resistance monitoring in ocular microorganisms (ARMOR) surveillance study. JAMA Ophthalmol 133: 1445-1454.

11. Upadhyay MP, Karmacharya PC, Koirala S, Shah DN, Shakya S, Shrestha JK, Bajracharya H, Gurung CK, Whitcher JP, 2001. The Bhaktapur eye study: ocular trauma and antibiotic prophylaxis for the prevention of corneal ulceration in Nepal. Br J Ophthalmol 85: 388-392. 